\title{
Habituation in the polychaete Hesperonoë adventor
}

JAMES A. DYAL, I University of Waterloo, Waterloo, Ontario, Canada, and KENNILEE HETHERINGTON, Texas Christian University, Ft. Worth, Texas 76129

Scaleworms were trained 20 trials per day for 3 days to traverse a straight alley. Consistent and substantial response decrement was exhibited under both massed and spaced conditions. Although both receptor adaptation and effector fatigue may have contributed to the performance level, the data were interpreted to reflect primarily a process of central habituation.

Habituation (Clark, 1960a, b) and instrumental discrimination learning (Evans, 1963; Flint, 1965), but not classical conditioning (Evans, 1966a, b, c), have been demonstrated in errant polychaetes of the family Neridae. It is not unlikely that substantial behavioral differences may exist among families of polychaetes because of the considerable differences which exist in brain development: It was the purpose of the present experiment to investigate the behavior of a representative of the family Polynoidae in an instrumental learning situation. The Ss were Hesperonoe adventor, a scaleworm which is commensal with the echiuroid Urechis caupo.

Since pilot work indicated that Hesperonoe adventor, like most polychaetes, is negatively phototaxic, it was felt that a bright light could be reinforced by a darkened goal box. Although Evans (1963) had demonstrated that entry into a darkened area could serve as a reinforcing event in the learning of an avoidance response in a $T$-maze, no evidence existed concerning the efficacy of this event to decrease locomotor speeds of polychaetes. It thus seemed that three possible results might be obtained under the boundary conditions of the present experiment: (1) Worms which were "rewarded" by a darkened area might exhibit a decrease in running time similar to that typically found with rats in a straight alley. Control worms, for which the goal box was not darkened, would show little or no response increment or perhaps some response decrement; (2) the darkened area might not constitute a reinforcing event and thus there would be no performance difference between experimental and control worns: and (3) the performance level of the experimental groups might be superior to that of the control group but both groups might exhibit response decrement due to habituation, receptor adaptation or effector fatigue.

\section{METHOD}

The Ss were 21 Hespernoe adientor which were selected at random from a larger group of 30 , with the restriction that no animal less than $1 \mathrm{in}$. in length be used. The mean length was $2 \mathrm{in}$. and the range was $1 \mathrm{in}$. to $3 \mathrm{in}$. They began training 2 to $48 \mathrm{~h}$ after capture and were not fed during the course of the experiment. Between experimental sessions they were housed in the dark in individual 6 in. diameter petri dishes in which the sea water was changed daily.

The apparatus consisted of a glass aquarium $1 \frac{1 / 2}{\mathrm{in}}$. wide with a start area demarcated by a black line and a Plexiglas goal box which was $5 \frac{1 / 4}{4}$ in. long, 1 in. wide, and 1 in. high. The floor of the goal box was beveled to a thickness of $1 \mathrm{~mm}$ at the entrance. The runway was illuminated by two desk lamps containing bulbs of $65 \mathrm{~W}$ and $25 \mathrm{~W}$ and a tensor lamp (GE, 93T). The two desk lamps were placed on each side of the runway about $7 \mathrm{in}$. from the start area, and 5 in. above the alley. The tensor lamp was placed 3 in. in front of the goal box and 3 in. above. It was possible to arrange the lamps in such a way that the goal box could have the same illumination as the runwaly or it could be darkened. Timing was done by means of stop watches.

The Ss were assigned at random to two treatment conditions. The experimental $\mathrm{Ss}$ were permitted to enter the darkened goal box on every trial whereas the control Ss were required to enter the illuminated goal box.

On the first trial of each day's training a worm was poured from the petri dish into the goal box. The goal box was then placed into the apparatus and the worm was washed into the start area. If it was facing the back of the start box, it was gently turned around with a camel hair brush. The $S$ was permitted to remain undisturbed in the start area for $30 \mathrm{sec}$. If it had not crossed the start line after this period it was gently touched on the posterior setae with the brush. Timing was begun when $S$ crossed the start line. Two time measures were taken: running time (RT), which was the time required to traverse the runway from the start line to the entrance to the goal box, a distance of 18 in., and goal time (GT), which was the time required from the time the $S$ touched the goal box entrance until he passed a point $21 / 4$ in. into the goal box. On each trial the $S$ was permitted to remain in the goal box for $60 \mathrm{sec}$. The goal box was then removed from the goal area to the start area, the $S$ was washed into the start area and a new trial was begun. The intertrial interval averaged about $5 \mathrm{sec}$. Twenty trials were run each day. The water in the aquarium was emptied after each worm was trained and the aquarium was refilled with fresh sea water to a depth of $1 / 2 \mathrm{in}$. The time of day when the worms were trained was counterbalanced over treatment conditions.

Two important procedural criteria were established: (1) a maximum of $180 \mathrm{sec}$ was allowed for swimming from the start line into the goal box on each trial. If a $S$ exceeded that time, he was given a time score of $180 \mathrm{sec}$ on GT (or GT and RT if he had not reached the goal box) and was gently brushed to initiate the running response. He was permitted to remain the usual $60 \mathrm{sec}$ in the goal box. (2) If a worm proved to be quite unresponsive to the lighted conditions in the runway, it was discarded from the experiment after meeting a criterion of 15 successive trials on Training Day 1 or 2 in which RT or GT was over $180 \mathrm{sec}$. A total of seven worms were discarded prior to the completion of training on Day 2, leaving a total of 14 .

\section{RESUITS}

The mean RTs for each group are plotted in blocks of five trials for Training Days 1-3. It may be seen that the performance level of the experimental group is consistently faster than that of the control group. On Day 1 the differences between the two groups in running time was not significant; on Day ? the difference approached significance $(U=4$; $\mathrm{p}=.095$ ) while on Day 3 the difference was highly significant $(\mathrm{U}=0 ; \mathrm{p}=.008)$. Analysis of the mean RT over all three acquisition days also revealed highly reliable differences between the experimental and control groups $(U=0$; $p=.008$ ). Analysis of the GT scores resulted in no reliable differences between groups on any day.

The most striking feature of the RT data represented in Fig. $l$ is that both Group $E$ and Group $C$ manifest substantial response decrement within each experimental session. When the data for all groups are combined over Days 1-3 and a comparison is made between performance on Trial Block 1 and Trial Block 4, only one worm ran faster in Block 4 than it did in Block 1 (and this was a matter of only 1 sec). Application of the binomial sign test to these differences resulted in $x=1 ; p=.001$. It is thus clear from the graphic representation and the statistical analysis that the overall response decrement is both consistent and substantial.

The obtained response decrement is, of course, suggestive of the process of central habituation. However, in order to interpret the effect as habituation both receptor adaptation and effector fatigue must be eliminated as prime explanatory constructs. Under the assumption that decay of receptor 


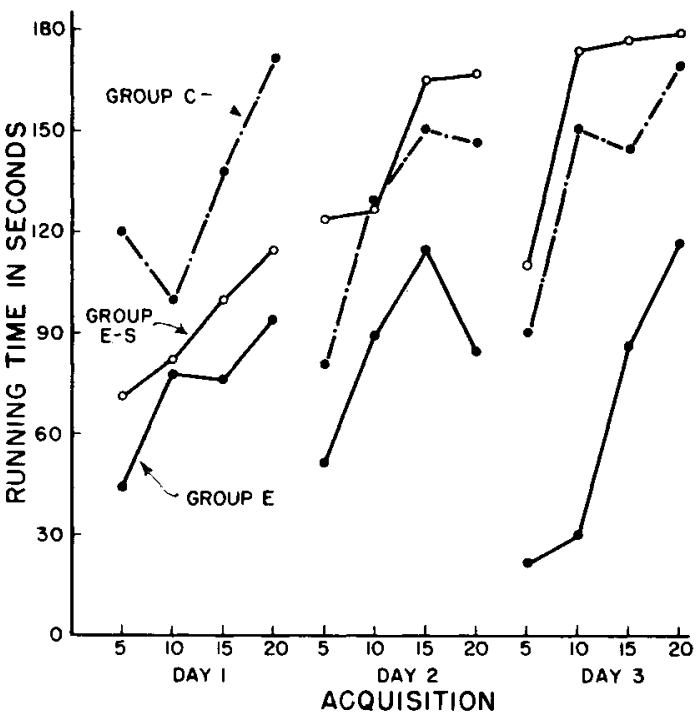

Fig. 1

adaptation and effector fatigue follows a more rapid time course than central habituation, it would be expected that increasing the length of the intertrial interval would result in less response decrement if either of these two processes were the prime determiners of the effect. Thus a third group of five worms was randomly selected from the $S$ pool. They were trained under the same conditions as Group $E$ except that, rather than running all 20 of the training trials in immediate succession, the trials were run in blocks of two. The average interval between the two-trial blocks was $54 \mathrm{~min}$. The interval between Trial 1 and Trial 2 of a block was about 5 sec. The interblock interval was spent in a plastic vial lying in the home petri dish. It may be seen from Fig. 1 that Group E-S exhibited considerable response decrement within each daily training session and that the magnitude of the decrement increased over training days. A comparison of RTs on Day 3 with those of Day 1 revealed no case in which a worm ran faster on Day $3(x=0 ; p=.03)$. It may also be seen that the difference between Group E-S and Group E increased over days. On Day 1 the groups were not reliably different but on Days 2 and 3 the differences were significant $(\mathrm{U}=2 ; \mathrm{p}=.032$; $\mathrm{U}=0 ; \mathrm{p}=.008)$. These results are just the opposite of what would be expected if receptor adaptation or effector fatigue were the prime determiners of the response decrement.

\section{DISCUSSION}

With regard to the several alternative results which could have been obtained, Alternative 3 most clearly describes the present data. The level of performance of Group $E$ was consistently faster than that of Group $C$ and all groups exhibited within-day response decrement. Although it is tempting to interpret these differences in level of performance as being due to the reinforcing effects of entry into a darkened goal box, the validity of such an explanation is questionable in the light of the fact that the observed group differences occur quite early in training. The mean RTs on Trial 2 of Day 1 were 39 and $145 \mathrm{sec}$ for Groups $\mathrm{E}$ and $\mathrm{C}$, respectively. It seems quite unlikely that one trial learning was occurring in an organism this low in the phylogenetic scale. Similar early effects of differential exposure to light in a straight alley have been reported in $L$. terrestris by Ratner (1964a). Because of these early differences in performance it is not possible to conclude from the data that entering a darkened goal box constitutes a reinforcing event for $H$. adventor. On the other hand, the data obviously should not be interpreted to say that learning based on entry into a darkened goal box cannot be demonstrated in $H$. adventor under other experimental conditions. For example, if it had been feasible to conduct the experiment so that the time-in-the-goal-box parameter was $5 \mathrm{~min}$ instead of $1 \mathrm{~min}$ perhaps a response increment rather than a decrement would have occured (Evans, 1963).

The clearest and most unequivocal finding of the present experiment is the consistant and substantial response decrement which was exhibited by all groups in the daily training sessions. Although both receptor adaptation and effector fatigue may have contributed to the absolute level of performance of each group these factors are not the prime determiners of the response decrement. On the contrary, the data support the interpretation that the response decrement reflects the process of central habituation. The argument for habituation can be further strengthened by reference to three collateral phenomena which have been cited among the common characteristics serving as a "... detailed operational definition of habituation ..." (Thompson \& Spencer, 1966). These are spontaneous recovery, dishabituation and potentiation. Spontaneous recovery may be seen in Fig. 1. Dishabituation was evidenced by vigorous locomotion elicited by light stimulation of the posterior setae with the camel hair brush. Potentiation, or a more rapid response decrement over training days, is clearly apparent in Group E and Group E-S. Neither receptor adaptation nor effector fatigue would be expected to show potentiation.

\section{REFERENCES}

CLARK, R. B. Habituation of the polychaete Nereis to sudden stimuli. I. General properties of the habituation process. Animal Behaviour, 1960 a, 8, 82-91.

CLARK, R. B. Habituation of the polychaete Nereis to sudden stimult II. Biological significance of habituation. Animal Behaviour, $1960 \mathrm{~b}, 8$, 92-103.

EVANS, S. M. Behavior of the polychaete Nereis in T-mazes. Animal Behaviour, 1963, 11, 379-393.

EVANS, S. M. Non-associative avoidance learning in nereid polychaetes. Animal Behaviour, 1966a, 14, 102-106.

EVANS, S. M. Non-associative behavioral modifications in the polychaete Nereis diversicolor. Animal Behaviour, 1966b, 14, $107-112$.

EVANS, S. M. Non-associative behavioral modifications in nereid polychaetes. Nature, 1966c, 211, 945-948.

FLINT, P. The effect of sensory deprivation on the behaviour of the polychaete Nereis in T-mazes. Animal Behaviour, 1965, 13, 187-193.

RATNER, S. C. Worms in a straight alley: Acquisition and extinction or phototaxis. Psychological Record, 1964, 14, 31-36.

THOMPSON, R. F., \& SPENCER, W. A. Habituation: A model phenomenon for the study of neuronal substrates of behavior. Psychological Review, 1966, 73, 16-43.

$$
\text { NOTE }
$$

1. This experiment was conducted at Bodega Marine Laboratory of the University of California during the first author's tenure as National Science Foundation Science Faculty Fellow. The authors wish to thank Dr. Cadet Hand, Director of the laboratory, for making the facilities available and Dr. Charles Stasek and Dr. Milton Miller for providing for collection of the specimens. 